\title{
British Medical Bulletin
}

Sickness presenteeism at work: prevalence, costs and management

\begin{tabular}{|c|c|}
\hline Journal: & British Medical Bulletin \\
\hline Manuscript ID & BMB-2018-006.R1 \\
\hline Manuscript Type: & Invited Review \\
\hline $\begin{array}{r}\text { Date Submitted by the } \\
\text { Author: }\end{array}$ & $\mathrm{n} / \mathrm{a}$ \\
\hline Complete List of Authors: & Kinman, Gail; University of Bedfordshire, Psychology \\
\hline Keywords: & \\
\hline
\end{tabular}

SCHOLARONE
Manuscripts 
Sickness presenteeism at work: prevalence, costs and management

\author{
Dr. Gail Kinman
}

Professor of Occupational Health Psychology

School of Psychology

University of Bedfordshire

Luton

Bedfordshire LU1 3JU

Gail.kinman@beds.a.cuk 
Sickness presenteeism at work: prevalence, costs and management

\begin{abstract}
Introduction: Presenteeism is defined as continuing to attend work during illness. As a growing health concern, awareness of the factors that encourage presenteeism and the risks of this behaviour is needed.
\end{abstract}

Sources of data: A narrative review of research obtained via several databases, including Medline and Psycinfo, was conducted.

Areas of agreement: A range of contextual and individual factors is associated with presenteeism. Workers in some sectors, such as healthcare, appear to be at greater risk. Presenteeism may facilitate rehabilitation and recovery but it can exacerbate existing health problems and increase the risk of subsequent illness and absence as well as impair workability.

Areas of controversy: The incidence of sickness presenteeism is rising, alongside reductions in absenteeism. The growing awareness of the costs of presenteeism, especially in safetycritical environments, suggests that it should be considered a risk-taking behaviour and carefully measured and managed.

Growing points and areas for developing research: Measuring presenteeism as well as absenteeism will provide more accurate information about employee health. Raising awareness of the risks of working while sick and the economic, moral, cultural and social pressures on employees to do so appears crucial. Systemic interventions to manage presenteeism based on research evidence are required.

Keywords: presenteeism; occupational health; rehabilitation; work-related stress 
Presenteeism

Presenteeism has been defined in several ways, but it most commonly refers to situations where people continue to work while unwell and not functioning to their full capacity $^{1}$. Evidence is growing that the incidence and costs of presenteeism are higher than absenteeism ${ }^{2,3}$, but investigating it is considerably more challenging. Although most organisations have processes to measure and manage sickness absence ${ }^{4}$, presenteeism is an 'invisible behaviour' as recording the prevalence and costs would be time-consuming and costly. There is currently no 'gold standard' for measuring presenteeism. Some studies have used single items to identify the proportion of people who report having worked while sick in a specified timescale, whereas others have assessed their propensity to work during illness ${ }^{3,5}$. The Stanford Presenteeism Scale ${ }^{6}$, one of the most widely-used measures, takes a more functional approach by evaluating the extent to which health problems can impair workers' cognitive, emotional and behavioural performance. Measures of presenteeism are essentially subjective, however, as they rely on employees' evaluations of their fitness for work, attitudes to taking time off sick and perceptions of reduced productivity, whereas studies using objective evaluations of health status and functioning are rare. The challenges of identifying the direct and indirect costs of sickness presenteeism are also acknowledged.

The prevalence of presenteeism

Attempts have been made to estimate the prevalence of presenteeism via self-report surveys. In 2010, the European Working Conditions Survey ${ }^{7}$ reported that $40 \%$ of respondents $(40,000$ people in 34 countries) had worked while they were sick for at least one day in the previous twelve-month period. Presenteeism, defined as working during illness on at least two days during the last year, was reported by $36.3 \%$ of men and $40.4 \%$ of women. 
The prevalence ranged from $23 \%$ (in countries such as Italy, Portugal and Poland) to above $50 \%$ (in countries such as Montenegro, Malta and Denmark).

Information on the extent of sickness absence is more readily available than presenteeism. In 2016, the UK Office for National Statistics (ONS) ${ }^{8}$ estimated that 137.3 million working days were lost to sickness and injury, with the average yearly absence estimated at 6.3 days per employee ${ }^{4}$. An annual survey of absence management conducted in the UK by the Chartered Institute of Personnel and Development ${ }^{8}$ (CIPD) indicated that sick leave is higher in larger organisations, in the public sector and among manual workers ${ }^{4}$. The most common reasons for short-term absence (classified as up to four-weeks) reported by organisations who responded to this survey were minor illness (such as colds, flu and migraine $=95 \%$ ), musculoskeletal problems (such as back pain and repetitive strain injuries $=$ $44 \%$ ), recurring medical conditions (such as asthma and angina $=31 \%)$ stress $(47 \%)$ and mental health problems (such as depression and anxiety $=34 \%$ ). Almost a quarter of respondents $(24 \%)$ indicated that non-genuine ill health was the most common cause of shortterm sickness absence in their organisation. Long-term absence (classified as four weeks or more) was most commonly caused by stress (29\%) acute medical conditions (23\%), mental ill-health $(13 \%)^{8}$.

The overall level of sickness absence in the UK has declined since $2003^{8}$. This does not mean that the population is becoming healthier, as there is evidence that people have become more likely to work during illness. The CIPD survey discussed above ${ }^{4}$ found that around one-third of the organisations who participated reported an increase in sickness presenteeism from the previous year. These organisations typically had longer average working hours and were almost twice as likely to report a rise in mental health problems among their staff. Organisations with higher rates of presenteeism also tended to have 
The causes of presenteeism

Whether people continue to work during illness will be influenced by several factors such as the type of disorder or injury, the degree of incapacitation, the extent to which they feel able to discharge their duties, and their personal sick record ${ }^{3,11}$. The health-related causes of presenteeism could be categorised under four headings: acute illnesses (such as colds, allergies and gastrointestinal problems), recurring complaints (such as allergies), chronic conditions (such as arthritis, musculoskeletal disorders, common mental health problems and insomnia) and lifestyle factors (such as physical inactivity, poor diet and smoking) $3,11,12,13$. The most common reasons why people work while unwell, however, are similar to those that lead to sickness absence: musculoskeletal disorders, gastrointestinal symptoms and common mental health problems ${ }^{3,12,13}$. Studies typically report a dose-response gradient, in that the number of health risk factors and the frequency of illness factors will increase the probability that people will work while sick ${ }^{14}$. The duration of the health problems is also important, as chronic disorders or disability (particularly mental health problems) may be considered a less legitimate cause of sickness absence by patients and organisations ${ }^{9}$.

Several demographic and contextual determinants of presenteeism have also been identified. Some studies have found a slightly higher prevalence among women ${ }^{7,15,16,17}$, while others have found no gender differences ${ }^{11}$. While sickness absence may be more 
legitimised in female-dominated workplaces, there is some evidence that when organisational and individual difference factors (such as working hours, responsibilities, job commitment and stress) are controlled, any gender differences become non-significant ${ }^{12}$. The reasons that underpin sickness presenteeism may differ, however, between men and women ${ }^{35}$. In terms of age, some studies have found that presenteeism is more common among young to middleaged workers, presumably due to stronger attendance requirements by more junior staff ${ }^{12}$. Nonetheless, there is also evidence that taking time off to recover from minor illness is considered less legitimate in people with more senior roles in organisations ${ }^{18}$. Research findings also suggest that people with line management responsibilities are more likely to work while sick as they feel they need to set an example to their staff ${ }^{18}$. Evidence for employment status as a risk factor for presenteeism is mixed, but there is some evidence that the prevalence is greater in professional and highly-skilled white-collar workers ${ }^{4,7}$.

The rates of sickness absence for self-employed people are consistently lower than those who work for others ${ }^{8}$. A recently-published study that followed a representative sample of 100,000 people working in Portugal for over four years found that self-employment was associated with an $85 \%$ lower risk of sick leave than waged workers ${ }^{19}$. Such findings could be interpreted in several ways. Healthy people may be more likely to be self-employed, those in poorer health may return to waged work (or drop out of employment entirely), or the increased autonomy associated with working for oneself may be genuinely salutogenic. Nonetheless, there is evidence that self-employed workers are more likely to work while sick than those who are waged ${ }^{20}$. This may be due to lack of sick pay, feeling indispensable and being responsible for the livelihood of their staff. 'Self-employment' is a broad category, however, and embraces the growing number of people working in the 'gig' economy. Future research should examine sickness absence behaviours in this sector where work may be 
flexible, but is typically precarious, competitive and low-paid. Under such conditions working while sick may be particularly common and risky for health.

Other determinants of sickness presenteeism have been highlighted in the literature. Economic factors, such as job insecurity, a lack of alternative employment options and limited entitlement to sick pay, appear to be strong motivators to continue to work while sick 1,17. Under such conditions, there is some evidence that employees (especially those who are older) may engage in presenteeism to 'save up' their paid sick leave for future illness that may be more debilitating ${ }^{18}$. The rise in casual contracts may be at least partially responsible for the diminishing levels of absenteeism and rising presenteeism highlighted above, as people may feel obliged to work while sick if they have no other source of income ${ }^{21}$. This argument is supported by evidence that sickness absence rates increase and sickness presenteeism decreases when individuals move from insecure to secure employment where entitlement to paid sick leave is more likely ${ }^{22}$.

Specific working conditions have also been linked with presenteeism. Robust attendance management policies that operate trigger-point systems (where employees may lose pay, be subjected to disciplinary action, or even dismissed after a threshold level of absence is reached) are common causes of working during sickness, especially for people with chronic conditions ${ }^{23,17}$. Organisations frequently use absence as a key performance indicator ${ }^{4}$ which can discourage people from taking sick leave in order to enhance their promotion prospects and increase the likelihood of continued employment. Features of work, such as high demands, heavy workloads, time pressure, shift-work and long working hours, are also key predictors of presenteeism ${ }^{24,25,17}$. People with low job control, particularly those who are less able to modify their work and delegate to others, also typically report working while sick more frequently ${ }^{11}$ 
What has been termed 'individual boundarylessness' (or difficulty saying 'no' to requests from others) can increase the likelihood of working while sick ${ }^{18}$. It is plausible that other individual difference factors, such as conscientiousness, may also discourage people from taking time off sick but little research has yet been conducted. Considerably more evidence has been found for the influence of the social environment at work in shaping sick leave behaviours. Cooperation, loyalty and mutual respect among colleagues can encourage people to continue to attend work while unwell ${ }^{18}$, but other studies have found that supportive relationships at work can discourage presenteeism ${ }^{17}$. Workplace cultures where managers and colleagues role-model healthy sickness behaviours and provide adequate cover may offer reassurance that taking sick leave is the most appropriate action ${ }^{18}$.

Positive orientations towards work, such as intrinsic motivation, organisational commitment and feelings of fulfilment and satisfaction, can encourage sickness presenteeism ${ }^{17}$. Feelings of self-efficacy have also been identified as an important predictor of continuing to attend work during periods of ill health in people with chronic illness ${ }^{25}$. A recent study of more than 6,000 academic employees in UK universities found that engagement (comprising vigour and dedication to work) was one of the strongest predictors of working while sick during the previous year (along with work demands and lack of support $)^{25}$. Job satisfaction is beneficial for health and can enhance recovery processes, but the long-term risks of over-commitment and a reluctance to disengage from work have also been identified ${ }^{26}$. This suggests that people who engage in presenteeism may have short-term health benefits, but their wellbeing may deteriorate over time.

Presenteeism in healthcare professionals

Although there is evidence that sickness presenteeism is rising in general, it is particularly common among so-called 'helping' professionals. Healthcare workers, more 
specifically, have many of the recognised risk factors for presenteeism discussed above: the work is demanding, jobs can be highly specialised, working hours are often long and antisocial, and employees are at high risk of stress and burnout ${ }^{1,3,27}$. Moreover, healthcare professionals typically have a strong sense of duty and moral obligation for the welfare of others that can increase the pressure to attend work ${ }^{16}$, especially under current conditions where staffing levels are low and care is rationed ${ }^{28}$.

The prevalence and costs of presenteeism have been examined in different healthcare settings such as nursing, midwifery, care work, and pharmacy ${ }^{16,28,29}$. There is evidence that physicians are particularly likely to work while sick, with recent NHS statistics showing that doctors take a third fewer sick days than other healthcare workers ${ }^{30}$. Research conducted in several countries has examined the rates of presenteeism among doctors and the antecedents and consequences of such behaviour. Studies in the UK during the 1990s highlighted the propensity of working while sick in physicians ${ }^{31,32}$. More recently, a survey of 1,806 senior practitioners working in New Zealand's public health system found that nearly nine out of ten participants $(88 \%)$ had attended work while being too sick to meet their usual standards of performance over the previous two-year period ${ }^{33}$. Three-quarters of the sample disclosed that they had worked while experiencing infectious illness. A recent study of 536 US clinicians also found that more than eight out of ten $(83 \%)$ had worked while they were unwell ${ }^{34}$. More than half indicated that they would continue to attend work despite showing signs of potentially infectious disease, such as respiratory symptoms and diarrhoea, although most (95\%) recognised that this put patients at risk. The risk that working while unwell can pose to others, especially in safety-critical roles, is discussed further below.

Research findings indicate that healthcare professionals engage in presenteeism for several reasons. Feelings of duty and commitment to patients appear to be particularly 
important, but several other factors have been cited such as lack of cover, concerns about patients' continuity of care, fear of letting colleagues down and losing their respect, and pressure to portray a 'healthy' image ${ }^{34,35}$. These studies indicate that strong professional and cultural norms of altruism and even self-sacrifice are commonplace in healthcare and taking sick leave is often considered a sign of weakness. Doctors, in particular, may resist taking on a patient role and self-treatment is common, both of which can encourage working while sick 31,35. More knowledge is needed about healthcare workers' decision-making processes when they experience ill-health and the factors that encourage them to work while sick or to absent themselves. Insight into the cognitions they use to balance the risks and benefits to patients, colleagues and their own wellbeing when deciding to attend or refrain from work is of would be of particular value.

The impact of presenteeism - individual health outcomes

It is clear that presenteeism is not necessarily damaging and working while not fully fit can be therapeutic. The companionship and self-esteem that comes from purposeful activity can aid recovery, provide distraction from minor symptoms, and encourage people to reject the sick role. A study of over 2,000 workers with musculoskeletal disorders who were referred to a functional restoration programme support this argument, as those who continued to attend work were more likely to complete the programme, return to work to full-duty or full-time, and to remain in work one year after treatment ${ }^{36}$. The extent to which working conditions, such as duties and working hours, can be adjusted to meet employees' needs is a key factor in determining whether presenteeism facilitates or impairs recovery ${ }^{13}$. Different health complaints result in different limitations that, in turn, will require different adjustments. For example, musculoskeletal problems can constrain physical functioning and 
depression can engender difficulties with time management, whereas both complaints can impair cognitive and interpersonal skills ${ }^{37}$.

Although working while sick can facilitate recovery, evidence for its potentially damaging effects for the wellbeing of employees and others is growing. Several high-quality prospective studies and a recent systematic review concluded that presenteeism increases the risk of future health problems and long-term sickness absence ${ }^{38}$. A cohort study of 1,831 Japanese employees found that sickness presenteeism measured at baseline was associated with depression and absence for mental health problems a year later, after controlling for age and gender ${ }^{39}$. Moreover, a cohort of 5,071 male civil servants from the Whitehall II study ${ }^{40}$ were subjected to baseline screening of health status and coronary risk factors and their sickness absence records were analysed over the following three years. Results showed that 'unhealthy' people that took no sick leave during the follow-up period had double the risk of serious coronary events of unhealthy employees whose sickness absence was moderate. There is evidence that even minor illnesses can eventually become serious and lead to extended absenteeism if ignored ${ }^{41}$. Chronic work-related stress and emotional exhaustion have also been positively associated with working while sick ${ }^{17}$. Over time, stress and burnout can also increase the risk of many health problems, such as coronary heart disease, hypertension and depression, and down-grade the immune system ${ }^{42}$.

The impact of presenteeism on others

Many studies have highlighted the negative effects of working while sick on productivity at work $^{1,6,1}$; this is a particular risk for employees with comorbid physical and mental health complaints ${ }^{37}$. The risks of presenteeism for the health and safety of other people have also been recognised. Attending work when experiencing contagious illness 
(known as contagious presenteeism) is a recognised public health hazard that is likely to be particularly harmful when interacting with vulnerable people ${ }^{43}$. Presenteeism also has more indirect risks for the wellbeing of other people. Employees may be required to put more effort into their work to accommodate the compromised performance of sick colleagues, thereby increasing their own work demands and associated threats to health as well as potentially reducing their ability to take sick leave. More research is needed into the systemic influences and effects on sickness absence behaviours in organisations that can extend the knowledge gained about individual factors.

Most studies that have considered the implications of presenteeism for others have been conducted in healthcare environments. There is evidence that working while sick can lead to emotional exhaustion that can encourage cynical and depersonalising attitudes towards patients ${ }^{27}$, with potentially serious consequences. For example, analysis of data from 178 matched pairs of physicians and hospitalised patients found that doctors' depersonalisation resulted in lower patient satisfaction and longer post discharge recovery time ${ }^{44}$. Presenteeism has also been associated with an increased risk of errors and accidents in healthcare contexts ${ }^{45}$. A study of UK pharmacists found that they were more likely to prescribe the wrong medication or dosage when they were unwell ${ }^{29}$. Illness can impair job functioning, as more cognitive effort is needed to meet the required standards while simultaneously managing the distracting symptoms of illness. The capacity to monitor and respond promptly to environmental demands is also diminished during sickness, encouraging people to rely more heavily on routine and increasing the risk of errors and lapses of judgement. Moreover, fatigue (a symptom or outcome of many health conditions) can impair perception, judgement and motor skills and is a major cause of accidents and 'near misses' in safety critical work such as aviation ${ }^{46}$. These findings highlight the potentially serious implications of working while sick for the wellbeing and safety of others but, as they 


\section{Managing presenteeism}

Although presenteeism can be beneficial, this article has highlighted its potential negative effects - especially for people whose work is safety critical. Most organisations are taking steps to reduce absenteeism ${ }^{4}$ but accomplishing this without increasing damaging presenteeism will not be easy. As discussed above, people work while sick for many economic, cultural, moral and social reasons that may be largely unrelated to their actual health status and functional limitations. These risk factors will interact; for example, an employee who is overloaded at work and whose job is insecure may be more likely to work during illness if they have a poor sick record. Moreover, healthcare professionals may be more likely to engage in presenteeism if their unit is short-staffed, they are deeply committed to the wellbeing of their patients and their line manager is reluctant to take time off sick when required.

People commonly find it difficult to decide whether sick leave is justified unless they are given explicit guidance from professionals: i.e. 'told' to either stay home or go to work. Having a medical diagnosis is likely to increase the perceived legitimacy of taking time off sick. A study that interviewed 30 people who had been absent for musculoskeletal complaints 
found that those with a specific medical diagnosis (such as a fracture) felt that their absenteeism was justified, whereas those with unidentifiable disorders (such as low back pain) found the decision to take sick leave more challenging ${ }^{47}$. Without clear guidelines some employees may 'play it safe' and absent themselves whereas others with a similar condition will continue to work, although their long-term health and the safety of others may be compromised. It is nonetheless challenging for medical professionals to establish with any certainty when, or to what extent, work will facilitate or hinder recovery from an illness, injury or a long-term condition for any individual worker. Indeed, research findings show that doctors themselves frequently work while sick because they lack clear guidelines from their employers on when they should take sick leave ${ }^{33}$. 'Visibility' of illness seems an important criterion for healthcare staff, as the study found that doctors often feel obliged to 'present' themselves at work to show others that they are genuinely ill before going off sick. Insight was also provided by the study into the perceived legitimacy of different complaints as a reason for doctors to take sick leave. Findings showed that fatigue, even if extreme, would not be considered legitimate, although its potentially serious effects on job performance (highlighted above) were acknowledged.

Multi-level, systemic approaches to managing presenteeism are required. Such initiatives should encompass primary prevention, where risks factors are identified and wherever possible eliminated at source, as well as secondary or individual-level interventions. An annual survey of UK human resource professionals discussed earlier in this article shows that many organisations see presenteeism as a growing cause for concern, as the proportion of participating organisations that take preventative action rose from $31 \%$ in 2015 to $48 \%$ in $2016^{4}$. Nonetheless, respondents to the survey acknowledged that operational demands frequently took precedence over the health and wellbeing of their employees and the overall level of work-related stress (a key predictor of presenteeism) reported remained 
high. It is crucial to encourage organisations to take a long-term view, where fears about the immediate costs of sickness absence are balanced by a recognition of the risks of presenteeism for the future health and functioning of employees. Undertaking a critical review of sickness management policies to ensure that staff are not penalised for taking sick leave should prove effective. Establishing cultural norms that encourage workers to take sufficient time off sick to recover also seems crucial. The behaviour of line managers is a key predictor of the sickness culture in organisations. Encouraging supervisors to help employees keep work in perspective and act as role models for appropriate and 'healthy' sickness behaviour is therefore likely to be particularly effective $e^{3,48}$.

Staff wellbeing surveys are other primary tools that can help organisations record trends in health complaints and associated attendance behaviours. As highlighted in this article, relying on sickness absence statistics to assess health status is misleading, so including a measure of sickness presenteeism will provide a more accurate indicator. Incorporating measures of health-related productivity loss, such as the Stanford Presenteeism Scale $^{6}$, and cross-referencing these findings with health and safety records would also communicate the risks of working while sick. This scale could be supplemented by openended questions that seek to identify the reasons why people work while sick. A preventative approach should also identify the structural factors that can underpin presenteeism discussed above, such as long working hours, high demands, low support and lack of sickness cover, using valid risk assessment procedures such as the UK Management Standards approach ${ }^{25,49}$.

There is evidence that providing opportunities for flexible working may reduce presenteeism, especially for people with long-term conditions ${ }^{50}$. The findings of a nested study of employees with rheumatoid arthritis support this view, as those who could selfschedule their working hours reported lower presenteeism and less activity impairment ${ }^{51}$. Flexible working arrangements enable an increasing number of people to work from home 
using mobile technologies. Employees who 'e-work' tend to have lower levels of sickness absence $^{52}$, presumably because they are better able to pace themselves and accommodate the limitations of any symptoms they may experience. Nonetheless, there is some evidence that technology can increase rather than reduce sickness presenteeism by encouraging employees to be 'always on'. Technology can enable people to continue to work at home (or even when hospitalised) despite being formally signed off sick; this is a particular risk when workloads are high, support and staffing levels are low, and employees are deeply involved in their work and miss the intellectual stimulation it provides ${ }^{53}$. Organisations may overlook or even implicitly encourage such behaviour, but it is likely to delay recovery and may even breach the employer's duty of care.

Interventions are particularly important in healthcare organisations where working during sickness may satisfy short-term operational imperatives but could have long-term risks for staff and patients. In such environments, it is particularly important to reframe taking legitimate sick leave as responsible and healthy behaviour and presenteeism as a potential public health risk. Of some concern are the findings of a recent study of healthcare workers in Croatia showing that staff in organisations with a stronger safety culture were more, rather than less, likely to work while sick ${ }^{54}$. These findings suggest that safety culture may over-ride the self-care of health professionals which will actually compromise rather than improve patient safety.

Occupational health physicians have a key role to play in providing guidance to organisations and employees on what constitutes 'fitness for work' and communicating the risks of presenteeism. They can also help employers introduce procedures to identify whether continuing to work is likely to enhance or impair recovery and how reasonable adjustments could be made. Working while not fully recovered could be part of a negotiated return-towork plan that may involve part-time or flexible arrangements, or tasks that are less 


\section{References}

1. Johns G. Presenteeism in the workplace: A review and research agenda. J Org Behav 2010; 31(4):519-42.

2. Centre for Mental Health. Managing Presenteeism: A Discussion Paper. Centre for Mental Health, 2011) [Online]. Available from: http://www.centreformentalhealth.org.uk/managing-presenteeism (accessed 4.2.18).

3. Garrow V. Presenteeism: A review of current thinking. Institute for Employment Studies (2016) Available from: http://www.employmentstudies.co.uk/system/files/resources/files/507_0.pdf (accessed 9.2.18).

4. Chartered Institute of Personnel and Development, CIPD Absence Management: Annual Survey Report, 2016. London: CIPD. Available from: 
https://www.cipd.co.uk/Images/absence-management_2016_tcm18-16360.pdf(accessed 4.2.18).

5. Gerich, J. Determinants of presenteeism prevalence and propensity: Two sides of the same coin, Arch Environ Occup Health, 2016; 3(4): 189-98.

6. Koopman C, Pelletier K R, Murray J F, Sharda C E, Berger M L, Turpin R S, Hackleman P, Gibson P, Holmes D M, Bendel T. Stanford presenteeism scale: health status and employee productivity, J Occup Environ Med, 2002; 44(1):14-20

7. Eurofound. Health and Wellbeing at Work: A Report Based on the Fifth European Working Conditions Survey. European Foundation for the Improvement of Living and Working Conditions. Available from:

http://www.eurofound.europa.eu/pubdocs/2013/02/en/1/EF1302EN.pdf (accessed 9.2.18).

8. Office for National Statistics. Available online: https://www.ons.gov.uk/employmentandlabourmarket/peopleinwork/labourproductivity/a

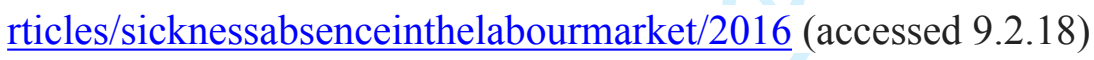

9. Miraglia M, Johns G. Going to work ill: A meta-analysis of the correlates of presenteeism and a dual-path model. J Occup Health Psych, 2016; 21(3):261.

10. Whysall A, Bowden J, Hewitt M. Sickness presenteeism: measurement and management challenges. Ergonomics 2018; 61(3): 341-54.

11. Schultz AB, Edington DW. Employee health and presenteeism: a systematic review. $J$ Occup Rehab 2007; 17(3):547-79.

12. Gosselin E, Lemyre L, Corneil W. Presenteeism and absenteeism: Differentiated understanding of related phenomena. J Occup Health Psych 2013; 18(1):75-86. 
13. Roe RA, Van Diepen B. Employee health and presenteeism: A challenge for human resources management. In: A Alexander-Stamatios, Cooper C (eds) New Directions in Organizational Psychology and Behavioral Medicine London: Routledge, 2011.

14. Goetzel RZ, Long, SR, Ozminkowski RJ, Hawkins K, Wang SH, Lynch W. Health, absence, disability, and presenteeism cost estimates of certain physical and mental health conditions affecting US employers. J of Occup Environ Med 2004; 46(4): 398412.

15. Sendén MG, Schenck-Gustafsson K, Fridner A. Gender differences in Reasons for Sickness Presenteeism-a study among GPs in a Swedish health care organization. Ann Occup Environ Med, 2016; 28(1):50-7.

16. Aronsson G, Gustafsson K, Dallner M. Sick but yet at work. An empirical study of sickness Presenteeism. J Epidemiol Community Health, 2000; 54:502-09

17. Miraglia M, Johns G. Going to work ill: A meta-analysis of the correlates of presenteeism and a dual-path model. J Occup Health Psychol, 2016; 21(3): 261-83.

18. Kinman G, Clements AJ, Hart J. When are you coming back? Presenteeism in UK prison officers. The Prison Journal (in press)

19. Goncalves J, Martins PS. The Effect of Self-Employment on Health: Evidence from Longitudinal Social Security Data. Available online at: http://ftp.iza.org/dp11305.pdf (Accessed: 9.2.18).

20. Johansen V, Aronsson G, Marklund S. Positive and negative reasons for sickness presenteeism in Norway and Sweden: a cross-sectional survey. BMJ Open, 2014; 4(2): e004123.

21. Kim JY, Lee J, Muntaner C, Kim SS. Who is working while sick? Nonstandard employment and its association with absenteeism and presenteeism in South Korea. Int Arch Occup Environ Health, 2016; 89(7):1095-101. 
22. Virtanen M, Kivimäki M, Elovainio M, Vahtera J, Ferrie JE. From insecure to secure employment: changes in work, health, health related behaviours, and sickness absence. Occup Env Med, 2003; 60(12):948-53.

23. Munir F, Yarker J, Haslam C. Sickness absence management: encouraging attendance or 'risk-taking' presenteeism in employees with chronic illness? Disabil Rehabil, 2008; 30(19): 1461-72.

24. Hansen CD, Andersen JH. Going ill to work-What personal circumstances, attitudes and work-related factors are associated with sickness presenteeism? Soc Sci Med 2008; 67(6):956-64.

25. Kinman G, Wray, S. Presenteeism in academic employees: occupational and individual factors. Occup Med, 2018; https://doi.org/10.1093/occmed/kqx191

26. Buddeberg-Fischer B, Klaghofer R, Stamm M, Siegrist J, Buddeberg C. Work stress and reduced health in young physicians: prospective evidence from Swiss residents. Int Arch Occup Environ Health, 2008; 82(1):31-8.

27. Demerouti E, Le Blanc PM, Bakker AB, Schaufeli WB, Hox J. Present but sick: a three-wave study on job demands, presenteeism and burnout. Career Dev Int, 2009; 14(1):50-68.

28. Dhaini SR, Zúñiga F, Ausserhofer D, Simon M, Kunz R, De Geest S, Schwendimann R. Are nursing home care workers' health and presenteeism associated with implicit rationing of care? A cross-sectional multi-site study. Geriatr Nurs, 2017; 38(1):33-8.

29. Niven K, Ciborowska N. The hidden dangers of attending work while unwell: A survey study of presenteeism among pharmacists. Int J Stress Manage, 2015; 22(2):207-21.

30. NHS Sickness Absence Rates, 2015. http://digital.nhs.uk/catalogue/PUB17903 (Accessed 9.2.18) 
31. McKevitt C, Morgan M, Dundas R, Holland WW. Sickness absence and 'working through' illness: a comparison of two professional groups. J Public Health, 1997; 19(3):295-300.

32. Forsythe M, Calnan M, Wall B. Doctors as patients: postal survey examining consultants and general practitioners' adherence to guidelines. BMJ, 1999; 319(7210):605-8.

33. Chambers C, Frampton C, Barclay M. Presenteeism in the New Zealand senior medical workforce - a mixed-methods analysis. New Zeal Med J, 2017; 130(1449): 10 .

34. Szymczak JE, Smathers S, Hoegg C, Klieger S, Coffin SE, Sammons JS. Reasons why physicians and advanced practice clinicians work while sick: a mixed-methods analysis. JAMA paediatrics, 2015; 169(9):815-21.

35. Senden MG, Løvseth LT, Schenck-Gustafsson K, Fridner A. What makes physicians go to work while sick? A comparative study of sickness presenteeism in four European countries (HOUPE). Swiss Med Wkly, 2013; 143:13840.

36. Howard KJ, Mayer TG, Gatchel RJ. Effects of presenteeism in chronic occupational musculoskeletal disorders: stay at work is validated. J Occup Environ Med, 2009; (6):724-31.

37. Burton N W, Pransky G, Conti D J, Chen C, Edington D W. The association of medical conditions and presenteeism. J Occup Environ Med, 2004; 46:38-45

38. Skagen J, Collins AM. The consequences of sickness presenteeism on health and wellbeing over time: A systematic review. Soc Sci Med, 2016; 169-77

39. Suzuki T, Miyaki K, Song Y, Tsutsumi A, Kawakami N, Shimazu A, Takahashi M, Inoue A, Kurioka S. Relationship between sickness presenteeism (WHO-HPQ) with 
depression and sickness absence due to mental disease in a cohort of Japanese workers. J ASffect Disord, 2015; 180:14-20.

40. Kivimäki M, Head J, Ferrie J E, Hemingway H, Shipley M J, Vahtera J, Marmot M G Working while ill as a risk factor for serious coronary events: the Whitehall II study. Am J Public Health, 2005; 95:98-102

41. Bergström G, Bodin L, Hagberg J, Aronsson G, Josephson M. Sickness presenteeism today, sickness absenteeism tomorrow? A prospective study on sickness presenteeism and future sickness absenteeism. J Occup Environ Med, 2009; 51(6):629-38.

42. Lovallo WR. Stress and Health: Biological and Psychological Interactions, 2015; California: Sage.

43. Widera E, Chang A, Chen HL. Presenteeism: a public health hazard. J Gen Intern Med, 2010; 25(11):1244-7.

44. Halbesleben J, Rathert C. Linking physician burnout and patient outcomes: Exploring the dyadic relationship between physicians and patients. Health Care Manag Rev, 2008; 33(1): 29-39

45. Letvak SA, Ruhm CJ, Gupta SN. Nurses' presenteeism and its effects on self-reported quality of care and costs. Am J Nurs, 2012; 112(2):30-8.

46. Wiegmann DA, Shappell SA. A Human Error Approach to Aviation Accident Analysis: The Human Factors Analysis and Classification System. Routledge; 2017

47. Hooftman WE, Westerman MJ, Van Der Beek AJ, Bongers PM, Van Mechelen W. What makes men and women with musculoskeletal complaints decide they are too sick to work? Scand J Work Environ Health, 2008;107-12.

48. Gilbreath B, Karimi L. Supervisor behavior and employee presenteeism. Int J Leader Studies, 2012; 7(1):114-31. 
49. MacKay CJ, Cousins R, Kelly PJ, Lee S, McCAIG RH. 'Management Standards' and work-related stress in the UK: Policy background and science. Work Stress, 2004; 18(2):91-112.

50. Irvine A. Fit for work? The influence of sick pay and job flexibility on sickness absence and implications for presenteeism. Social Pol Admin, 2011; 45(7):752-69.

51. Bansback N, Zhang W, Walsh D, Kiely P, Williams R, Guh D, Anis A, Young A. Factors associated with absenteeism, presenteeism and activity impairment in patients in the first years of RA. Rheumatol 2011; 51(2):375-84.

52. Noonan MC, Glass JL. The hard truth about telecommuting. Monthly Lab. Rev, 2012; 135:38.

53. Kinman G, Wray S. Presenteeism in academics: A shift to the twilight zone. 2017; HRZone. Available at https://www.hrzone.com/lead/culture/presenteeism-inacademics-a-shift-to-the-twilight-zone

54. Brborović H, Brborović O. Patient safety culture shapes presenteeism and absenteeism: a cross-sectional study among Croatian healthcare workers. Arch Indust Hyg Toxicol, 2017; 68(3):185-9. 


\section{Dr. Gail Kinman}

Professor of Occupational Health Psychology

School of Psychology

University of Bedfordshire

Luton

Bedfordshire LU1 3JU

Gail.kinman@beds.a.cuk 
Sickness presenteeism at work: prevalence, costs and implications: the costs and challenges

Abstract

Introduction: Presenteeism is defined as continuing to attend work while ill. As a growing public health concern, awareness of the factors that encourage presenteeism and the risks of this behaviour is required.

Sources of data: A narrative review was conducted based on a search was conducted of several databases, including Medline and Psycinfo.

Areas of agreement: $\underline{\text { A range of contextual and individual factors has been associated with }}$ presenteeism. Under some conditions presenteeism may enhance-facilitate rehabilitation and recovery, but it can exacerbate existing health problems and, increase the risk of subsequent illness and absence, and threaten the wellbeing of others. Presenteeism is particularly eommon among healtheare professionals and has the potential to threaten public health and safety.

Areas of controversy: The incidence of presenteeism is-appears to be rising alongside reductions in absenteeism. It is a particular risk factor for healthcare professionals. The growing awareness of the costs of presenteeism, especially in safety-critical environments such as healtheare, suggests that it should be perceived as a potential risk-taking behaviour that needs to be measured and managed.

Growing points and areas for developing research: Measuring sickness presenteeism as well as absenteeism will provide more accurate information about employee health. It is important to increase awareness of the risks of working while sick and the economic, moral, cultural and social pressures on employees to do so. Highlighting the wide-ranging costs of 
presenteeism will strengthen the case for recognising and managing such behaviour.

Occupational health professionals have a key role to play in this process. How presenteeism can be managed effectively is a key priority.

Keywords: presenteeism; occupational health; rehabilitation; work-related stress

Presenteeism

Presenteeism has been defined in several ways, but it most commonly refers to situations where people continue to work while unwell and not functioning to their full capacity ${ }^{1}$. Evidence is growing that the incidence and costs of presenteeism are considerably higher than absenteeism ${ }^{2,3}$, but investigating it systematically is more challenging. Although most organisations have attendance management policies and processes to measure and manage sickness absence ${ }^{4}$, there are several reasons why employees may be reluctant to disclose that they are working while sick and-recording such behaviourpresenteeism is likely to be time-consuming and costly as employers would be required to record the number of sickness presence days and/or a propensity for their staff to work while sick. Attempts have been made to estimate the prevalence of presenteeism in different countries via self-report surveys. There is currently no 'gold standard' for measuring presenteeism. Some studies have used single items to identify the proportion of people who report having worked while sick in a specified timescale, whereas others have assessed workers' tendencies to work during illness ${ }^{3,5}$. The Stanford Presenteeism Scale ${ }^{6}$, one of the most widely-used measures, takes a $\underline{\text { more functional approach by evaluating the extent to which health problems can impair }}$ workers' cognitive, emotional and behavioural performance at work. Measures of presenteeism are essentially subjective, as they rely on employees' personal evaluations of 
their fitness for work, attitudes to taking time off sick and perceptions of reduced

\section{The prevalence of presenteeism}

Attempts have been made to estimate the prevalence of presenteeism via self-report surveys. In 2010, the European Working Conditions Survey ${ }^{\mathbb{}}$ found that $40 \%$ of respondents (40,000 people in 34 countries) had worked while they were sick for at least one day in the previous twelve-month period. Presenteeism, classified as working during illness on at least two days during the last year, was reported by $36.3 \%$ of men and $40.4 \%$ of women. The prevalence of presenteeism ranged from 23\% (in countries such as Italy, Portugal and Poland) to above 50\% (in countries such as Montenegro, Malta and Denmark). - Information on the extent of sickness absence is more readily available. In 2016, the UK Office for National Statistics (ONS) ${ }^{86}$ estimated that 137.3 million working days were lost to sickness and injury (4.3 days per worker)and the average level of absence has been estimated at 6.3 days per $\underline{\text { employee }^{4}}$. An annual survey of absence management conducted in the $\mathrm{UK}^{4}$ by the Chartered Institute of Personnel and Development ${ }^{8}$ indicated that levels of sick leave areIt is higher in larger organisations, in the public sector and among manual workers 4 . In this $\underline{\text { survey, } \mathrm{t} \text { The most common reasons for short-term absence (classified as up to four-weeks) }}$ reported by organisations for all employees are-were minor illness (such as colds, flu and migraine $=95 \%$ ), musculoskeletal problems (such as back pain and repetitive strain injuries = $\underline{44 \%}$ ), recurring medical conditions (such as asthma and angina $=31 \%) \underline{\text { stress }}$ stress $\underline{(47 \%)}$ and mental health problems (such as depression and anxiety $=34 \%$ ). Almost a quarter of respondents (24\%) reported that non-genuine ill health was the most common cause of sickness absence in their organisation. Long-term absence (classified as four weeks or more) 

ill-health (13\%).

The overall level of sickness absence in the UK has declined since $2003^{86}$. This does not mean that the population is becoming healthier; there is evidence that people have become more likely to work during illness. The findings of an annual survey of organisations in the UK conductedthe survey discussed above by the Chartered Institute of Personnel and Development ${ }^{44}$ found-reported that around one-third reported an increase in sickness presenteeism from the previous year. These organisations tended to have longer working hours and were almost twice as likely to report a rise in mental health problems among their staff. Organisations with higher rates of presenteeism also tended to report increased absenteeism, suggesting that employees alternate both behaviours to manage episodes of illness. This supports the findings of a recent meta-analysis that reported strong positive correlations between absenteeism and presenteeism ${ }^{9}$. The pattern of absence and presence will differ according to health condition ${ }^{10}$, but also depend on a range of organisational and individual factors.

The causes of presenteeism

Whether people engage in presenteeism will depend on a wide rangebe influenced by several effactors such as the type of disorder or injury, the degree of incapacitation, and the extent to which they feel able to discharge their duties ${ }^{11}$. The health-related causes of presenteeism have beencould be categorised under four headings: acute illnesses (such as colds, allergies and gastrointestinal problems), recurring complaints (such as allergies), chronic conditions (such as arthritis, musculoskeletal disorders, common mental health 
problems and insomnia) and lifestyle factors (such as physical inactivity, poor diet and smoking) $)^{3,11,12,13}$. The most common reasons why people work while unwell, however, are similar to those that lead to sickness absence: musculoskeletal disorders, gastrointestinal symptoms and common mental health problems $\underline{3,12,13}$. Studies typically report a doseresponse gradient, in that the number of health risk factors and the frequency of illness factors increase the probability that people will work while sick $^{14}$.

Demographic and contextual determinants of presenteeism have also been identified. Some studies have found a slightly higher prevalence among women $7.15,16,17$, while others have found no gender differences ${ }^{1 \underline{1}}$. There is evidence ${ }_{2} \cdot \underline{h}$ However, that when organisational and individual difference factors (such as working hours, responsibilities, job commitment and stress) are controlled, any gender differences become non-significant $\frac{12}{2}$. Evidence for employment status and qualifications as risk factors for presenteeism is mixed, but some studies have found that the prevalence is greater in professional and highly-skilled whitecollar workers $\frac{4.7}{}$. Research findings also suggest that people with line management responsibilities are more likely to work while sick as they feel they need to set an example to their staff 18 .

$\underline{\text { The rates of sickness absence for people who are self-employed are consistently lower }}$ than those who are employed by others ${ }^{8}$. A recently-published study that followed a representative sample of 100,000 people working in Portugal for over four years found that self-employed was associated with an $85 \%$ lower risk of sick leave than waged workers ${ }^{19}$. These findings could be interpreted in several ways. Healthy people may be more likely to be self-employed, those in poorer health may return to waged work (or drop out of employment entirely), or the increased autonomy associated with working for yourself may be genuinely salutogenic. Nonetheless, there is evidence that self-employed workers are more likely to 
work while sick than those who are waged ${ }^{20}$. This may be due to several factors, such as lack of sick pay, as well as feeling indispensable and responsible for the livelihood of their staff. The category 'self-employment' is broad, however, and increasingly includes those working within the 'gig' economy. Future research should examine presenteeism in this sector where work is flexible, but typically precarious, competitive and low-paid.

Other determinants of presenteeism have been highlighted. Economic factors, such as job insecurity, a lack of alternative employment options and limited entitlement to sick pay, encourage people to work while sick ${ }^{1,17}$. The rise in casual contracts may be at least partially responsible for the diminishing levels of absenteeism and rising presenteeism highlighted $\underline{\text { above, as people may have little alternative but to work while sick if they have no alternative }}$ source of income ${ }^{21}$. This argument is supported by evidence that sickness absence rates increase and presenteeism decreases when individuals move from insecure to secure employment where they are more likely to be entitled to paid sick leave $\mathrm{e}^{22}$.

$\underline{\text { Specific working conditions have also been linked with presenteeism. Robust }}$ $\underline{\text { attendance management policies that operate trigger-point systems (where employees may }}$ lose pay, be subjected to disciplinary action, or even dismissed after a threshold level of absence is reached) are one of the most common cause of working during sickness, especially for people with long-term conditions ${ }^{23,17}$. Organisations frequently use absence as a key performance indicator ${ }^{4}$ which can discourage people from taking sick leave to enhance their promotion prospects and ensure continued employment. High demands, heavy workloads, time pressure and long working hours are also key predictors of presenteeism ${ }^{24,25,17}$. The quality of working relationships and the availability of support also influences attitudes towards taking sick leave. Cooperation, loyalty and mutual respect among colleagues can encourage people to attend work while unwell ${ }^{18}$, but other studies have found that workplace 
support might discourage presenteeism ${ }^{17}$. Workplace cultures that role-model healthy $\underline{\text { sickness behaviours and provide adequate support may provide reassurance that taking sick }}$ leave is the most appropriate action.

Positive orientations towards work, such as intrinsic motivation, organisational commitment and feelings of fulfilment and satisfaction, have also been found to encourage presenteeism ${ }^{17}$. A study of more than 6,000 academic employees in UK universities found that engagement (comprising vigour and dedication to work) was one of the key predictors of working while sick over the previous year (along with work demands and lack of support) ${ }^{25}$. Enjoyment of work is beneficial for health and can enhance recovery processes, but the longterm risks of over-commitment and a reluctance to disengage from work have also been identified $^{26}$.

\section{$\underline{\text { Presenteeism in healthcare professionals }}$}

Presenteeism is particularly common among so-called 'helping' professionals who meet the primary needs of others on a daily basis. Healthcare workers are particularly likely to work while sick ${ }^{16,24}$. Such work has several recognised risk factors for presenteeism discussed above: the work is demanding, jobs can be highly specialised, working hours are often long and antisocial and employees are at high risk of stress and burnout ${ }^{1,3,2 \underline{17}}$. Moreover, healthcare professionals typically have a strong sense of duty and moral obligation for the welfare of others that can increase the pressure to attend work ${ }^{16}$, especially where staffing levels are low and care is rationed ${ }^{28}$.

The prevalence and costs of presenteeism has been examined in different healthcare settings such as nursing, midwifery, care work and pharmacy ${ }^{16,28,29}$. The prevalence of 
sickness presenteeism among $\mathrm{p}$ Physicians is are particularly highlikely to work while sick, with estimates between $80 \%$ and $90 \%{ }^{20}$. Recentwith recent NHS statistics indicatindicatinged that doctors take a third fewer sick days than other healthcare workers ${ }^{30}$. Research conducted in several countries has examined the rates of presenteeism among doctors in particular and the causes and consequences of such behaviour. Studies in the UK during the 1990s initially highlighted the incidence and risks of working while sick for physicians ${ }^{31,32}$. A survey of 1,806 senior practitioners working in New Zealand's public health system found that nearly nine out of ten participants ( $88 \%$ ) had attended work while being too sick to perform temeet their usual standards of performance over the previous two-year period ${ }^{33}$. Three-quarters of the sample disclosed that they had worked while experiencing infectious illness. A recent study of 536 US clinicians also found that more than eight out of ten (83\%) had worked while they were unwell ${ }^{34}$. More than half of the sample indicated that they would continue to attend work despite showing signs of potentially infectious disease such as respiratory symptoms and diarrhoea, although most (95\%) recognised that this put patients at risk. The risk that working while unwell can pose to others, especially in safety-critical professions, is discussed further below.

These studies indicateResearch findings indicate that doctors engage in presenteeism for several reasons. Feelings of duty and commitment to patients appears to be particularly common, but several other factors were cited such as low staffing levels, lack of cover, concerns about patients' continuity of care, fear of letting colleagues down and losing their respect, and the need to portray a 'healthy' image ${ }^{34,35}$. These studies indicate that sStrong professional and cultural norms of altruism and even self-sacrifice are commonplace and, where taking sick leave is often considered a risk to the profession and a sign of weakness. More knowledge is needed about physicians' decision-making processes when they experience ill-health and the factors that encourage them to work while sick or absent 
themselves. Insight into the cognitions they use to balance the risk to patients, colleagues and their own wellbeing and sense of professional efficacy of attending or not attendingwhen deciding to attend or refrain from work is of particular interest.

The impact of presenteeism_ individual health outcomes

It is important to recognise that presenteeism is not necessarily damaging and working while not fully fit can be therapeutic. The companionship and self-esteem that comes from purposeful activity can aid recovery, distract people from minor symptoms, and encourage them to move beyond the sick role. A study of over 2,000 workers with musculoskeletal disorders who were referred to a functional restoration programme found that those who continued to attend work were more likely to complete the programme, return to work to fullduty or full-time, and to remain in work one year after treatment ${ }^{365}$. The extent to which working conditions, such as duties and working hours, can be adjusted to meet employees' needs is aA key factor in determining whether presenteeism facilitates or impairs recovery is the extent to which working conditions, such as duties and working hours, can be adjusted to meet employees' needs ${ }^{13}$. Different health complaints will result in different limitations that, in turn, will require different adjustments. For example, musculoskeletal problems can constrain physical functioning and depression can engender difficulties with time management, while both complaints can impair cognitive and interpersonal skills ${ }^{37}$.

Although working while sick has some potential to facilitate recovery, evidence for its damaging effects on health and safety is growing. A recent systematic review and sseveral high-quality prospective studies have found that presenteeism increases the risk of future health problems and long-term sickness absence, $\frac{38}{2}$ after controlling for demographic and 
The impact of presenteeism on others

The risks of presenteeism for the health and safety of other people have also been recognised. Attending work when experiencing contagious illness (known as contagious presenteeism) is a recognised public health hazard that is likely to be particularly harmful when working with vulnerable people ${ }^{43}$. Presenteeism also has more indirect risks for the wellbeing of others. Colleagues may be required to put more effort into their work to accommodate the compromised performance of sick colleagues, thereby increasing their own work demands and associated threats to health. 
Most studies that have considered the implications of presenteeism for others have been conducted in healthcare settings. There is evidence that working while sick can lead to $\underline{\text { emotional exhaustion that can encourage cynical and depersonalising attitudes towards }}$ patients $^{27}$ with potentially serious consequences. For example, analysis of data from 178 matched pairs of physicians and hospitalised patients found that doctors' depersonalisation resulted in lower patient satisfaction and longer post discharge recovery time ${ }^{44}$ Presenteeism has also been associated with an increased risk of errors and accidents, as well as poorer ratings of care by patients ${ }^{45}$. A study of UK pharmacists found that they were more likely to prescribe the wrong medication or dosage when they were unwell ${ }^{29}$. Illness can impair job performance as more cognitive effort is needed to meet the standards required in the face of the distracting symptoms of illness. The capacity to monitor and respond promptly to environmental demands is also diminished, encouraging people to rely more heavily on routine thus increasing the risk of errors and lapses of judgement. Moreover, fatigue, a $\underline{\text { symptom of many health conditions, can impair perception, judgement and motor skills and }}$ is a major cause of accidents and "near misses ${ }^{46}$. These studies highlight the serious implications of working while sick for others but, as they typically rely on subjective reports of presenteeism and health and safety performance, more objective assessments are required to identify the risks. Research with longitudinal designs is also needed to identify the implications of presenteeism for the health and safety of other people over time.

\section{Managing presenteeism}

Although presenteeism is not necessarily harmful, this article has highlighted its potential negative effects especially for people whose work is safety critical. Most organisations are taking steps to reduce absenteeism ${ }^{4}$ but achieving this without increasing 
damaging presenteeism will be challenging. As discussed above, people work while sick for many economic, cultural, moral and social reasons that may be largely unrelated to their health status and functional limitations. These factors will also interact; for example, an employee who is overloaded at work and whose job is insecure may be more likely to work during illness if they have recently taken sick leave. Moreover, healthcare professionals may be more likely to engage in presenteeism if their unit is short-staffed, they are deeply committed to the wellbeing of their patients and their line manager also works through $\underline{\text { illness. }}$

There is evidence that people find it difficult to decide whether sick leave is justified unless they are given explicit guidance from professionals: i.e. 'told' to either stay home or to go to work. A study that interviewed 30 people who had been absent for musculoskeletal complaints found that those with a specific medical diagnosis (such as a fracture) felt that their absenteeism was justified, whereas those with unidentifiable disorders (such as low back pain) found the decision to take sick leave more challenging ${ }^{47}$. Without clear guidelines some employees may 'play it safe' and absent themselves while others with a similar condition will continue to work, although their long-term health and the safety of others may be compromised. It is nonetheless challenging for medical professionals to establish with any certainty when, or to what extent, work will facilitate or hinder recovery from an illness, injury or a long-term condition for any individual worker. Indeed, research findings show that doctors themselves frequently work while sick because they lack clear guidelines from their employers on when they should take sick leave ${ }^{33} \cdot$.'Visibility' of illness seems an important criterion for healthcare staff, as this study found that doctors often feel obliged to 'present' themselves at work to show demonstrate to others that they are genuinely ill before going off sick. Insight was also provided into the perceived legitimacy of different complaints as a reason for doctors to take sick leave. Findings showed that fatigue, even if extreme, would 
not be considered a legitimate reason for sickness absence, although its potentially serious effects on job performance (outlined above) was-were acknowledged.

Multi-level, systemic approaches to managing presenteeism are required. Such initiatives should include primary prevention, where risks factors are identified and wherever possible eliminated at source, as well as secondary or individual level interventions. An annual survey of UK human resource professionals shows that many organisations see presenteeism as a growing cause for concern,with and the proportion of participating organisations taking that take preventative action rising rose from $31 \%$ in 2015 to $48 \%$ in $2016^{4}$. Nonetheless, survey respondents acknowledged that operational demands frequently took precedence over the health and wellbeing of their employees and the overall level of work-related stress (a key predictor of presenteeism) reported remained high. It is crucial to encourage organisations to take a long-term view where fears about the immediate costs of sickness absence are balanced by a recognition of the risks of presenteeism for the future health and functioning of employees-and how it can undermine their professional functioning. Undertaking a critical review of sickness management policies to ensure that employees are not penalised for taking sick leave to manage their conditions should prove effective.

Establishing cultural norms that encourage people to take sufficient time off sick to recover is also erucialvital. The behaviour of line managers is a streng fundamental predictor of the sickness culture in organisations. Encouraging managers to help employees keep work in perspective and to act as role models for appropriate and 'healthy' sickness behaviour are- $\underline{\text { is }}$ likely to be particularly effective $e^{3,48}$.

Staff wellbeing surveys could help organisations record trends in health complaints and associated attendance behaviours $\underline{s}$ (both absenteeism and presenteeism). Relying on sickness absence statistics to assess health status is misleading, so including a measure of $\underline{\text { sickness presenteeism is likely to provide a more accurate indicator. Incorporating measures }}$ 
of health-related productivity loss, such as the Stanford Presenteeism Scale ${ }^{6}$, and crossreferencing these findings with health and safety records would also highlight the risks of working while sick. This could be supplemented by open-ended questions that seek to identify the reasons why people work while sick. A preventative approach should also identifyttention to the structural factors that can underpin presenteeism, such as long working hours, high demands, low support and lack of sickness cover, is alse required-using valid risk assessment procedures such as the UK Management Standards approach ${ }^{25,49}$. There is evidence that increasing opportunities for flexible working may reduce presenteeism, especially for people with long-term conditions ${ }^{50}$. The findings of a nested study of employees with rheumatoid arthritis support this view, as those who could self-schedule their working hours had lower presenteeism and activity impairment ${ }^{51}$. Flexible working arrangements allow an increasing number of people to work from home using mobile technologies. People who 'e-work' tend to have lower levels of sickness absence ${ }^{52}$, presumably because they are more able to accommodate the limitations of any symptoms they may experience. Nonetheless, there is some evidence that technology can facilitate damaging presenteeism by encouraging employees to be 'always on'. It can enable people to continue to work at home (or even when hospitalised) despite being formally signed off sick; this is a particular risk when workloads are high, support and staffing levels are low, and employees are deeply involved in their work and miss the intellectual stimulation it provides $^{53}$. Organisations may turn a blind eye tooverlook or even implicitly encourage such behaviour, but it is likely to delay recovery and may be eonsidered-a breach of the employer's duty of care.

Interventions are particularly important in healthcare organisations where working during sickness may satisfy short-term operational imperatives but could have long-term risks for staff and patients. In such environments, it is particularly important to reframe taking 
legitimate sick leave as responsible and healthy behaviour and presenteeism as a potential public health risk. Of some concern are the findings of aA recent study of healthcare workers in Croatia indicating that staff in organisations with a stronger safety culture were more, rather than less, likely to work while sick ${ }^{54}$. These findings indieate-suggest that safety culture can over-ride the self-care of health professionals which will in fact compromise rather than improve patient safety.

Occupational health physicians have a key role to play in providing guidance to organisations and employees on what constitutes 'fitness for work' and communicating the risks of presenteeism. They can also help employers introduce procedures to identify whether continuing to work is likely to enhance or impair recovery and how reasonable adjustments could be made. Working while not fully recovered could be part of a negotiated return-towork plan that may involve part-time or flexible arrangements, or tasks that are less demanding. Occupational health professionals could also help organisations reduce some of the risks for absenteeism and presenteeism by introducing secondary-level interventions aiming to improveing the general health and fitness of employees via wellness programmes and self-care guides. As stress has been associated with presenteeism, employee assistance programmes and multi-level stress management initiatives could also be effective.

Presenteeism is not a new phenomenon but, compared to absenteeism, it has been little studied. Knowledge of the factors that encourage people to work while sick and the implications for employees, organisations and members of the public is growing. The introduction of evidence-informed systemic interventions to reduce damaging presenteeism, especially in safety-critical environments, are therefore crucial. Physicians have a key role to 
play in building more healthy sickness absence cultures in organisations, both for their personal wellbeing and for the health and safety of others.

\section{References}

1. Johns G. Presenteeism in the workplace: A review and research agenda. J Org Behav 2010; 31(4):519-42.

2. Centre for Mental Health. Managing Presenteeism: A Discussion Paper. Centre for Mental Health, 2011) [Online]. Available from: http://www.centreformentalhealth.org.uk/managing-presenteeism (accessed 4.2.18).

3. Garrow V. Presenteeism: A review of current thinking. Institute for Employment Studies (2016) Available from: http://www.employment$\underline{\text { studies.co.uk/system/files/resources/files/507 } 0 . p d f}$ (accessed 9.2.18).

4. Chartered Institute of Personnel and Development, CIPD Absence Management: Annual Survey Report, 2016. London: CIPD. Available from: https://www.cipd.co.uk/Images/absence-management_2016_tcm18-16360.pdf(accessed 4.2.18).

5. Gerich, J. Determinants of presenteeism prevalence and propensity: Two sides of the same coin, Arch Environ Occup Health, 2016; 3(4): 189-98.

6. Koopman C, Pelletier K R, Murray J F, Sharda C E, Berger M L, Turpin R S, Hackleman P, Gibson P, Holmes D M, Bendel T. Stanford presenteeism scale: health status and employee productivity, J Occup Environ Med, 2002; 44(1):14-20

7. Eurofound. Health and Wellbeing at Work: A Report Based on the Fifth European Working Conditions Survey. European Foundation for the Improvement of Living and Working Conditions. Available from: 
http://www.eurofound.europa.eu/pubdocs/2013/02/en/1/EF1302EN.pdf (accessed 9.2.18).

8. Office for National Statistics. Available online: https://www.ons.gov.uk/employmentandlabourmarket/peopleinwork/labourproductivity/a

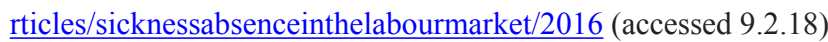

9. Miraglia M, Johns G. Going to work ill: A meta-analysis of the correlates of presenteeism and a dual-path model. J Occup Health Psych, 2016; 21(3):261.

10. Whysall A, Bowden J, Hewitt M. Sickness presenteeism: measurement and management challenges. Ergonomics 2018; 61(3): 341-54.

11. Schultz AB, Edington DW. Employee health and presenteeism: a systematic review. $J$ Occup Rehab 2007; 17(3):547-79.

12. Gosselin E, Lemyre L, Corneil W. Presenteeism and absenteeism: Differentiated understanding of related phenomena. J Occup Health Psych 2013; 18(1):75-86.

13. Roe RA, Van Diepen B. Employee health and presenteeism: A challenge for human resources management. In: A Alexander-Stamatios, Cooper C (eds) New Directions in Organizational Psychology and Behavioral Medicine London: Routledge, 2011.

14. Goetzel RZ, Long, SR, Ozminkowski RJ, Hawkins K, Wang SH, Lynch W. Health, absence, disability, and presenteeism cost estimates of certain physical and mental health conditions affecting US employers. J of Occup Environ Med 2004; 46(4): 398412.

15. Sendén MG, Schenck-Gustafsson K, Fridner A. Gender differences in Reasons for Sickness Presenteeism-a study among GPs in a Swedish health care organization. Ann Occup Environ Med, 2016; 28(1):50-7.

16. Aronsson G, Gustafsson K, Dallner M. Sick but yet at work. An empirical study of sickness Presenteeism. J Epidemiol Community Health, 2000; 54:502-09 
17. Miraglia M, Johns G. Going to work ill: A meta-analysis of the correlates of presenteeism and a dual-path model. J Occup Health Psychol, 2016; 21(3): 261-83.

18. Kinman G, Clements AJ, Hart J. When are you coming back? Presenteeism in UK prison officers. The Prison Journal (in press)

19. Goncalves J, Martins PS. The Effect of Self-Employment on Health: Evidence from Longitudinal Social Security Data. Available online at: http://ftp.iza.org/dp11305.pdf (Accessed: 9.2.18).

20. Johansen V, Aronsson G, Marklund S. Positive and negative reasons for sickness presenteeism in Norway and Sweden: a cross-sectional survey. BMJ Open, 2014; 4(2):e004123.

21. Kim JY, Lee J, Muntaner C, Kim SS. Who is working while sick? Nonstandard employment and its association with absenteeism and presenteeism in South Korea. Int Arch Occup Environ Health, 2016; 89(7):1095-101.

22. Virtanen M, Kivimäki M, Elovainio M, Vahtera J, Ferrie JE. From insecure to secure employment: changes in work, health, health related behaviours, and sickness absence. Occup Env Med, 2003; 60(12):948-53.

23. Munir F, Yarker J, Haslam C. Sickness absence management: encouraging attendance or 'risk-taking' presenteeism in employees with chronic illness? Disabil Rehabil, 2008; 30(19): 1461-72.

24. Hansen CD, Andersen JH. Going ill to work-What personal circumstances, attitudes and work-related factors are associated with sickness presenteeism? Soc Sci Med 2008; 67(6):956-64.

25. Kinman G, Wray, S. Presenteeism in academic employees: occupational and individual factors. Occup Med, 2018; https://doi.org/10.1093/occmed/kqx191 
26. Buddeberg-Fischer B, Klaghofer R, Stamm M, Siegrist J, Buddeberg C. Work stress and reduced health in young physicians: prospective evidence from Swiss residents. Int Arch Occup Environ Health, 2008; 82(1):31-8.

27. Demerouti E, Le Blanc PM, Bakker AB, Schaufeli WB, Hox J. Present but sick: a three-wave study on job demands, presenteeism and burnout. Career Dev Int, 2009; 14(1):50-68.

28. Dhaini SR, Zúñiga F, Ausserhofer D, Simon M, Kunz R, De Geest S, Schwendimann R. Are nursing home care workers' health and presenteeism associated with implicit rationing of care? A cross-sectional multi-site study. Geriatr Nurs, 2017; 38(1):33-8.

29. Niven K, Ciborowska N. The hidden dangers of attending work while unwell: A survey study of presenteeism among pharmacists. Int J Stress Manage, 2015; 22(2):207-21.

30. NHS Sickness Absence Rates, 2015. http://digital.nhs.uk/catalogue/PUB17903 (Accessed 9.2.18)

31. McKevitt C, Morgan M, Dundas R, Holland WW. Sickness absence and 'working through' illness: a comparison of two professional groups. J Public Health, 1997; 19(3):295-300.

32. Forsythe M, Calnan M, Wall B. Doctors as patients: postal survey examining consultants and general practitioners' adherence to guidelines. BMJ, 1999; 319(7210):605-8.

33. Chambers C, Frampton C, Barclay M. Presenteeism in the New Zealand senior medical workforce — a mixed-methods analysis. New Zeal Med J, 2017;

130(1449): 10 
34. Szymczak JE, Smathers S, Hoegg C, Klieger S, Coffin SE, Sammons JS. Reasons why physicians and advanced practice clinicians work while sick: a mixed-methods analysis. JAMA paediatrics, 2015; 169(9):815-21.

35. Senden MG, Løvseth LT, Schenck-Gustafsson K, Fridner A. What makes physicians go to work while sick? A comparative study of sickness presenteeism in four European countries (HOUPE). Swiss Med Wkly, 2013; 143:13840.

36. Howard KJ, Mayer TG, Gatchel RJ. Effects of presenteeism in chronic occupational musculoskeletal disorders: stay at work is validated. J Occup Environ Med, 2009; (6):724-31.

37. Burton N W, Pransky G, Conti D J, Chen C, Edington D W. The association of medical conditions and presenteeism. J Occup Environ Med, 2004; 46:38-45

38. Skagen J, Collins AM. The consequences of sickness presenteeism on health and wellbeing over time: A systematic review. Soc Sci Med, 2016; 169-77

39. Suzuki T, Miyaki K, Song Y, Tsutsumi A, Kawakami N, Shimazu A, Takahashi M, Inoue A, Kurioka S. Relationship between sickness presenteeism (WHO-HPQ) with depression and sickness absence due to mental disease in a cohort of Japanese workers. J ASffect Disord, 2015; 180:14-20.

40. Kivimäki M, Head J, Ferrie J E, Hemingway H, Shipley M J, Vahtera J, Marmot M G Working while ill as a risk factor for serious coronary events: the Whitehall II study. Am J Public Health, 2005; 95:98-102

41. Bergström G, Bodin L, Hagberg J, Aronsson G, Josephson M. Sickness presenteeism today, sickness absenteeism tomorrow? A prospective study on sickness presenteeism and future sickness absenteeism. J Occup Environ Med, 2009; 51(6):629-38.

42. Lovallo WR. Stress and Health: Biological and Psychological Interactions, 2015; California: Sage. 
43. Widera E, Chang A, Chen HL. Presenteeism: a public health hazard. J Gen Intern Med, 2010; 25(11):1244-7.

44. Halbesleben J, Rathert C. Linking physician burnout and patient outcomes: Exploring the dyadic relationship between physicians and patients. Health Care Manag Rev, 2008; 33(1): 29-39

45. Letvak SA, Ruhm CJ, Gupta SN. Nurses' presenteeism and its effects on self-reported quality of care and costs. Am J Nurs, 2012; 112(2):30-8.

46. Wiegmann DA, Shappell SA. A Human Error Approach to Aviation Accident Analysis: The Human Factors Analysis and Classification System. Routledge; 2017

47. Hooftman WE, Westerman MJ, Van Der Beek AJ, Bongers PM, Van Mechelen W. What makes men and women with musculoskeletal complaints decide they are too sick to work? Scand J Work Environ Health, 2008;107-12.

48. Gilbreath B, Karimi L. Supervisor behavior and employee presenteeism. Int J Leader Studies, 2012; 7(1):114-31.

49. MacKay CJ, Cousins R, Kelly PJ, Lee S, McCAIG RH. 'Management Standards' and work-related stress in the UK: Policy background and science. Work Stress, 2004; 18(2):91-112.

50. Irvine A. Fit for work? The influence of sick pay and job flexibility on sickness absence and implications for presenteeism. Social Pol Admin, 2011; 45(7):752-69.

51. Bansback N, Zhang W, Walsh D, Kiely P, Williams R, Guh D, Anis A, Young A. Factors associated with absenteeism, presenteeism and activity impairment in patients in the first years of RA. Rheumatol 2011; 51(2):375-84.

52. Noonan MC, Glass JL. The hard truth about telecommuting. Monthly Lab. Rev, 2012; $135: 38$. 
53. Kinman G, Wray S. Presenteeism in academics: A shift to the twilight zone. 2017; HRZone. Available at https://www.hrzone.com/lead/culture/presenteeism-inacademics-a-shift-to-the-twilight-zone

54. Brborović H, Brborović O. Patient safety culture shapes presenteeism and absenteeism: a cross-sectional study among Croatian healthcare workers. Arch Indust Hyg Toxicol, 2017; 68(3):185-9. 